\title{
UM MODELO DE DECISÃO \\ PARA PRIORIZAÇÃO NO PLANEJAMENTO DE SISTEMAS DE INFORMAÇÃO
}

Adiel Teixeira de Almeida, PhD

Professor - Universidade Federal de Pernambuco; Cx. Postal 7462 - Recife-PE; 50722-970; aalmeida@npd.ufpe.br, aalmeida@elogica.com.br

Palavras chave: apoio a decisão multicritério, planejamento de sistemas de informação, priorização de sistemas de informação

Key words: multicriteria decision support, information system planning, information system priorities

\section{RESUMO}

$O$ artigo trata de um modelo de decisāo para priorizaçāo de Sistemas de Informaçāo (SI). Esta priorizaçáo faz parte de um processo mais amplo de planejamento de SI. Neste planejamento os fatores estratégicos da organizaçāo e os aspectos de criticidade de processos sāo associados aos agrupamentos de informaçāo. A priorizaçăo dos módulos de SI considera os aspectos estratégicos e tático-operacionais dos processos utilizando uma função valor aditivo. Após uma apresentação da metodologia de planejamento de SI, segue-se uma descriçāo do modelo de decisâo para priorizaçāo dos módulos de SI. Para finalizar, um estudo de caso ilustra numericamente a aplicação do procedimento.

\section{ABSTRACT}

The paper describes part of a research concerned with the planning of Information Systems (IS). The prioritisation of the IS is emphasised. Strategic factors and critical aspects of processes are taken into acoount to obtaining the priority of the modules of IS. The organisation strategic planning and the analysis of the business process are applied to set priorities for the whole IS. The priorities of the IS modules are established based on preference modelling using an additive value function. Finally a case study based on

design of a particular Information System (IS) is presented, including output result for the case study. 


\section{PRODUÇÃO}

\section{1 - Introdução}

$\mathrm{O}$ artigo apresenta uma proposta para apoiar o processo decisório na priorização de Sistemas de Informação (SI), dentro de um contexto de Planejamento de SI, que inclui outros aspectos precedentes à etapa de priorização.

A metodologia de Planejamento de SI resulta de um conjunto de trabalhos anteriores (Almeida et al, 1992; Almeida et al, 1991) elaborados a partir do desenvolvimento de um projeto de pesquisa nesta linha. Aprimoramentos da metodologia referencial anterior foram obtidos na simplificação dos estágios de desenvolvimento do trabalho e especialmente na etapa de priorização (Almeida e Alcoforado, 1996a). A engenharia da informação utiliza na etapa de priorização do sistema de informação um protótipo de sistema de apoio a decisão, cujos resultados foram demonstrados com um estudo de caso (Almeida \& Alcoforado, 1996b).

A metodologia de Planejamento é iniciada através de um estudo da organização para se obter sua visão Estratégica. A partir da missão e da visão estratégica da organização é desenvolvida uma a nálise processual. Segue-se a Engenharia de Informação para obtenção da arquitetura do Sistema de Informação. A a rquitetura do sistema de Informação contempla, dentre outros aspectos, os
Módulos de Serviços de Informação (MSI's). O processo de priorizaçāo destes MSI's é baseado em agregação aditiva das preferências em relação aos critérios, incluindo principalmente a visão dos processos organizacionais, diretamente associados à Gestão Estratégica da Organização.

O Planejamento resulta num Plano de Ação que inclui Plano Diretor de Serviço de Informação, apresentando a linha estratégica a ser seguida para acompanhamento do caminho estratégico da organização, e incluindo ensaios dos novos desenvolvimento tecnológicos, visto ser um plano de longo prazo.

Assim, o artigo apresenta uma visão do Planejamento de Sistema de Informação, onde está inserido o problema de priorização, que é detalhado a seguir e ilustrado através de uma aplicação.

\section{2 - Metodologia para Planejamento do Sistema de Informação}

A metodologia aplicada para Planejamento de Sistema de Informação (Almeida \& Alcoforado, 1996a) é apresentada de forma resumida a seguir. $A$ figura 1 apresenta as etapas de desenvolvimento para construção da arquitetura do SI. 


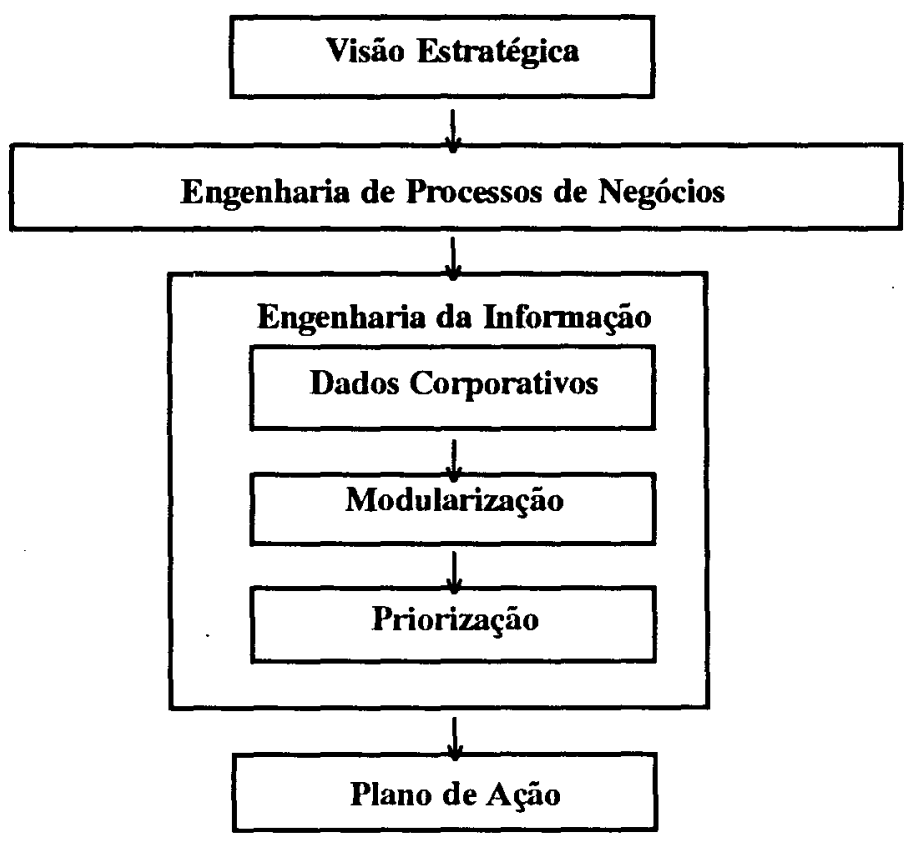

Figura 1 - Metodologia para Planejamento de Sistemas de Informação

A metodologia é iniciada através de um estudo da organização para se obter sua visão Estratégica, caso não estejam disponiveis os resultados de um processo de administração estratégica. Esta visão estratégica é sintetizada na forma de fatores estratégicos, objetivando orientar as ações estratégicas a serem desenvolvidas na organização.

Aos fatores Estratégicos são atribuídos pesos $\left(e_{i}\right)$. Considera-se a obtenção de $n$ fatores estratégicos, representados pelo vetor a seguir, de tal modo que $\sum_{i=1}^{n} e_{i}=1$, e $0 \leq e_{i} \leq 1$ :

$$
\left\|e_{i}\right\|=\left[\begin{array}{l}
e_{1} \\
\cdots \\
e_{n}
\end{array}\right]
$$

Após a explicitação da missāo e da visão estratégica da organizaçāo, é desenvolvida a Engenharia de Processos de Negócio que consiste da definição de todos os processos que são desenvolvidos nos vários setores da área estudada. Os 


\section{PRODUÇÃO}

processos são a estrutura pela qual uma organização faz o necessário para produzir valor para os seus clientes (Davenport, 1994).

Segue-se a Engenharia de Informação para obtenção da arquitetura do Sistema de Informação, que permitirá abordar a questão da informação a partir das classes de dados corporativos e dos processos de negócios da organização. A arquitetura do sistema de Informação contempla, dentre outros aspectos, os Agrupamentos de Informações (AGI's), e os diversos Tipos de tecnologias, ferramentas, que podem ser usadas para o tratamento destas. A combinação destas duas visões (AGI's e Tipos de tecnologias) forma os Módulos de Serviços de Informaçāo (MSI's). Esta fase da metodologia é composta por três etapas: Dados Corporativos, Modularização e Priorização.

Na primeira etapa, são definidas as entidades e as classes de dados, ou seja, os grandes grupos de dados corporativos da organização. A partir desta visão corporativa dos dados, sempre relacionados a um cliente/produto, passa-se à modularização. Nesta fase são obtidos os Agrupa mentos de Informação (AGI's). É aplicado um método de aglutinação, de modo que estes AGI's consistem em todas as informações, atendendo a um grupo de processos que tenham em comum, as mesmas classes de dados envolvidas. Além dos AGI's, o sistema é visualizado através dos Tipos de Serviços de Informação (TSI's). Esta sub-divisão consiste na abordagem, ferramenta ou tecnologia utilizada para tratar os dados. A combinação entre os AGI's e os TSI's fornece os Módulos de Serviços de Informação (MSI's).

Na fase de modularizaçāo, é construída a estrutura informacional da organização. Os módulos fornecem ferramentas de gestão, através de um agrupamento de informações, que suporta a execução de um conjunto de processos específicos da organização. Finalmente, esta fase se encerra com a Priorização destes Módulos, que tem o objetivo de fornecer um adequado e claro posicionamento e priorização das ações de investimentos, despesas e necessidades relativas ao desenvolvimento dos MSI's.

Na última etapa, obtém-se o Plano de Ação, que inclui o Plano Diretor de Serviço de Informação. Este Plano Diretor é o resultado do encadeamento das informaçōes obtidas nas fases anteriores $e$ consiste na apresentação das Diretrizes e Metas para desenvolvimento dos MSI's, baseadas nas prioridades estabelecidas. Estas prioridades, por sua vez, são baseadas nas necessidades identificadas pela Engenharia de Processos de Negócio, e nos objetivos a serem atingidos, estabelecidos pelo Plano Estratégico.

A apresentação do modelo de decisão para priorização requer melhor detalhamento da estrutura dos MSI's. A determinação dos Módulos de Serviços de Informação (MSI's) é estruturada a partir das duas visôes para a Informação. Ou seja, a patir de uma segmentação matricial das duas visões para se agrupar a informação (AGI e TSI).

Na primeira visão (AGI), agrupam-se informações pela sua natureza; mais especificamente, tenta-se segmentar as 
informaçōes relacionadas a processos considerando-se tabém classes de dados. Assim, todas as informaçóes que fornecessem capacidade de gestão para determinado(s) processo(s) tratando determinada(s) classe(s) de dado(s) formam um Agrupamento de Informação (AGI).

No segundo enfoque, caracteriza-se a forma de abordagem utilizada no tratamento da informação. É utilizado basicamente, o paradigma proposto por Sprague e Watson (1989), considerando as três abordagens básicas para sistemas de informação. As três abordagens consistem em: Sistemas Transaciona is, Sistemas de Informação Gerencial e Sistemas de Apoio a Decisāo (Sprague \& Watson, 1989; Bidigoli, 1989; Thierauf, 1982). Outras abordagens interessantes de se ressaltar são acrescentadas a estas três. Esta visão modificada e estendida define os Tipos de Serviços de Informaçâo (TSI's), ou seja, os tipos de recursos (ferramenta/tecnologia) utilizados para o tratamento da informação.
Assim, os AGI's dependem do contexto a nalisado, ou seja, dos processos e especialmente dos dados da organização. Já os TSI's são pré-fixados, ou seja, são os tipos de abordagens consideradas na visāo modificada do paradigma mencionado, a saber: TSIT (Sistema de Informação Transaciona); TSIG (Sistema de Informação Gerencial); TSAD (Sistema de Apoio a Decisão); TSIE (Sistema de Informação para Executivos); TSAE (Sistema de Apoio a Escritório); TSAP (Sistema de Automação da Produção); TSAG (Sistema Apoio a Composição Gráfica).

A modularização, então, consiste em cruzar cada AGI com cada um dos TSI's, obtendo-se assim os Módulos de Serviços de Informação (MSI's). Isto pode ser visualizado através de uma matriz, onde a cada coluna é associada um AGI e a cada linha, um TSI. Desta forma as células resultantes correspondem aos MSI's, conforme ilustra a Figura 2.

\begin{tabular}{l|l|l|l|l} 
& AGI1 & AGI2 & AGI3 & AGI4 \\
\hline TSIT & MSI11 & MSI21 & & MSI41 \\
\hline TSIG & MSI12 & & & \\
\hline TSAD & & & & \\
\hline TSIE & & & & \\
\hline TSAE & & & & \\
\hline TSAP & & & & \\
\hline TSAG & MSI7 & & & MSI44
\end{tabular}

Figura 2 - Processo de Obtenção dos MSI's 
Na priorização serāo obtidos pesos para os MSI's, que serão representados pela matriz a seguir, considerando m AGI's e r TSI's.:

$$
\left\|s_{i j}\right\|=\left[\begin{array}{cccc}
s_{11} & s_{12} & \ldots & s_{1 m} \\
s_{21} & & & \\
\ldots & & & \\
s_{r 1} & \ldots & \ldots & s_{r m}
\end{array}\right]
$$

\section{Modelo de Decisão para Priorização de MSI's}

A priorização é realizada através da distribuição de pesos para os diversos aspectos a serem considerados para os AGI's e TSI's. O desenvolvimento de um tipo de TSI possui aspectos favoráveis e dificuldades, que serão considerados através dos pesos atribuídos, os quais representam custo/benefício desta implementação. Já os AGI's são ponderados, principalmente sob a ótica dos processos atendidos, de acordo com vários aspectos que refletirāo sua importância relativa na organização.

O modelo de decisão para priorização é baseado na modelagem de preferências utilizando uma função valor aditivo (Keeney \& Raiffa, 1976; Vincke, 1992.). Assim, os diversos critérios são combinados através da funçāo a seguir, considerando-se uma situação sem incerteza e independência em preferência entre os critérios (Keeney \& Raiffa, 1976): valor da alternativa $\mathrm{j}=\sum_{i=1}^{n} v_{i j} w_{i}$

onde: $v_{1 \mathrm{j}}=$ valor da alternativa $\mathrm{j}$ sob a ótica do critério $i ; w_{j}=$ peso do critério $i ; n$ = número de critérios; consideram-se os valores numa escala normalizada de modo que $0 \leq \mathrm{w}_{1} \leq 1, \mathrm{e} \sum_{i=1}^{n} w_{i}=1$.

Os índices para o sistema de priorização são compostos por dois conjuntos que apresentam: a Visão dos AGI's, e a Visão dos TSI's. Os índices selecionados para análise dos AGI's incorporam a visão da Engenharia de Processos do Negócio, sobre os processos suportados pelo $\mathrm{AGI}$, no que se refere à aderência às estratégias da organização, ao grau e criticidade de automação e inclusive aos aspectos comportamentais das pessoas envolvidas. Já os índices que analisam os TSI's abordam os aspectos tecnológicos, de oportunidade de mercado, custo relativo e de facilidade de implantação.

Na primeira visão tem-se os índices relativos a FATORES DE PONDERAÇĀO DOS AGI'S. Na segunda visão tem-se os chamados FATORES DE PONDERAÇÃO DOS TSI'S.

Após a obtenção dos índices relativos aos fatores dos AGI's e dos TSI's, segue-se a obtenção dos pesos dos MSI's, que apresentam uma visão final da modularização a partir dos dois anteriores. 


\section{FATORES DE PONDERAÇĀO DOS} AGI'S

Estes fatores enfatizam as necessidades da organização no processo de priorização. São considerados $v$ fatores que são agregados de modo a compor o peso geral de cada AGI. Dentre os diversos fatores que podem ser agregados, nesta etapa inicial, considera-se $v=3$. Assim, são utilizados os seguintes:

- ie = índice de aderência estratégica

- ic = índice de criticidade de automação

- iu = índice de comprometimento do usuário

Estes três índices são detalhados a seguir.

- Indice de Aderência aos Fatores Estratégicos - Expressa o quanto os processos que compõem o AGI participam no desenvolvimento das diretrizes estratégicas.

Inicialmente é obtido o peso estratégico dos processos e através deste, o índice de aderência dos AGI's aos fatores estratégicos.

Consideram-se $\mathbf{p}$ processos e $\mathbf{n}$ fatores estratégicos. Define-se a matriz que estabelece uma relação entre os fatores estratégicos e os processos, de modo que

$$
\begin{aligned}
& \sum_{i=1}^{p} r_{i j}=1 ; \text { para qualquer } \mathrm{j}, \mathrm{e} \\
& 0 \leq r_{i j} \leq 1 .
\end{aligned}
$$

$$
\left\|r_{i j}\right\|=\left[\begin{array}{cccc}
r_{11} & r_{12} & \ldots & r_{1 n} \\
r_{21} & & & \\
\ldots & & & \\
r_{p 1} & \ldots & \ldots & r_{p n}
\end{array}\right]
$$

Assim, o peso estratégico dos processos é definido através do vetor:

$\left\|p_{i}\right\|=\left[\begin{array}{c}p_{1} \\ \cdots \\ p_{p}\end{array}\right]$,

e é dado pela relação:

$\left\|p_{i}\right\|=\left\|r_{i j}\right\| \cdot\left\|e_{j}\right\|=\left\|\sum_{j=1}^{n} r_{i j} e_{j}\right\|(1)$

de modo que $\sum_{i=1}^{p} p_{i}=1, \mathrm{e}$

$0 \leq p_{i} \leq 1$

Considera-se que o número de AGI'S é igual a $\mathrm{m}$. De forma similar, obtém-se o índice de aderência dos AGI's aos fatores estratégicos, representado pelo vetor:

$$
\left\|a e_{i}\right\|=\left[\begin{array}{c}
a e_{1} \\
\ldots \\
a e_{m}
\end{array}\right] .
$$

Inicialmente, obtém-se o grau de relação 


\section{PRODUÇÃO}

do AGI com o processo, definido pela matriz:

$$
\left\|r a_{i j}\right\|=\left[\begin{array}{cccc}
r a_{11} & r a_{12} & \ldots & r a_{1 p} \\
r a_{21} & & & \\
\ldots & & & \\
r a_{m 1} & \ldots & \ldots & r a_{m p}
\end{array}\right]
$$

de tal modo que $0 \leq r a_{i j} \leq 1$; e

$$
\sum_{i=1}^{m} r a_{i j}=1 \text {, para qualquer } \mathbf{j}
$$

Finalmente, o índice de aderência dos AGI's aos fatores estratégicos é obtido através da relação a seguir, de forma que

$$
\begin{gathered}
\sum_{i=1}^{m} a e_{i}=1, \text { e } 0 \leq a e_{i} \leq 1: \\
\left\|a e_{i}\right\|=\left\|r a_{i j}\right\| \cdot\left\|p_{j}\right\|=\| \sum_{j=1}^{p} r a_{i j} p_{j}
\end{gathered}
$$

- Índice de Comprometimento do Usuário - Representa a disposição e o interesse do usuário pelo desenvolvimento do AGI e é representado pelo vetor.

$$
\begin{aligned}
& \left\|a u_{i}\right\|=\left[\begin{array}{c}
a u_{1} \\
\cdots \\
a u_{m}
\end{array}\right], \text { de modo que } \\
& \sum_{i=1}^{m} a u_{i}=1 \text {, e } 0 \leq a u_{i} \leq 1 .
\end{aligned}
$$

Este índice é obtido através do nível de comprometimento do usuário com os $\mathrm{p}$ processos, representado pelo vetor.

$$
\begin{gathered}
\left\|u_{i}\right\|=\left[\begin{array}{c}
u_{1} \\
\ldots \\
u_{p}
\end{array}\right], \text { de modo que } \\
\sum_{i=1}^{p} u_{i}=1, \text { e } 0 \leq u_{i} \leq 1 .
\end{gathered}
$$

Assim, aui é obtido pela relação:

$$
\left\|a_{i}\right\|=\left\|r_{i j}\right\| \cdot\left\|u_{j}\right\|
$$

- Criticidade de Automacão representa o grau de criticidade dos processos envolvidos devido à não automação ou à automação parcial; indica a necessidade de automação do processo sob a ótica operacional. Agrega dois aspectos: o grau de automação e as necessidades de automação. Este índice é representado pelo vetor:

$$
\left\|a c_{i}\right\|=\left[\begin{array}{c}
a c_{1} \\
\cdots \\
a c_{m}
\end{array}\right] \text {, de modo que }
$$

$\sum_{i=1}^{m} a c_{i}=1$, e $0 \leq a c_{i} \leq 1$.

Este índice é obtido através do nível de criticidade dos p processos, representado pelo vetor: 


$$
\left\|c_{i}\right\|=\left[\begin{array}{c}
c_{1} \\
\cdots \\
c_{p}
\end{array}\right], \text { onde } \sum_{i=1}^{p} c_{i}=1
$$

e $0 \leq c_{i} \leq 1$.

Assim, aci é obtido pela relação:

$$
\left\|\mathrm{ac}_{\mathrm{i}}\right\|=\left\|\mathrm{ra}_{\mathrm{ij}}\right\| \cdot\left\|\mathrm{c}_{\mathrm{j}}\right\|
$$

\section{Peso Geral do AGI}

Os índices que representam os fatores de ponderação dos AGI'S são agregados de modo a se obter um índice geral do AGI, representado pelo vetor:

$$
\left\|a_{i}\right\|=\left[\begin{array}{c}
a_{1} \\
\cdots \\
a_{m}
\end{array}\right]
$$

Este resultado é obtido através da relação:

$$
\left\|a_{i}\right\|=i e\left\|a e_{i}\right\|+i c\left\|a c_{i}\right\|+i u\left\|a u_{i}\right\|
$$

onde, os índices são ponderados em importância de modo que ie $+\mathrm{ic}+\mathrm{iu}=1$. Assim tem-se $\sum_{i=1}^{m} a_{i}=1$, e $0 \leq a_{i} \leq 1$. Observa-se que pode-se adotar uma forma mais geral para os índices operacionais (ic, iu), agregando-os antes. Desta forma, seriam considerados nesta etapa apenas dois índices (aderência estratégica e o operacional). Outra solução mais geral consiste em adotar uma forma mais geral para a equação (5), que é apresentada a seguir.

\section{Generalização na Obtenção do Peso Geral do AGI}

Neste caso, considera-se a utilização de $v$ fatores de ponderação dos AGI's. Assim, para a obtenção do peso dos AGI's, considera-se $o$ índice relativo dos $\mathrm{v}$ fatores de ponderação dos AGI'S, representado pelo vetor a seguir, de modo que

$$
\begin{aligned}
\sum_{i=1}^{v} i a_{i} & =1, \text { e } 0 \leq i a_{i} \leq 1 . \\
\left\|i a_{i}\right\| & =\left[\begin{array}{c}
i a_{1} \\
\ldots \\
i a_{v}
\end{array}\right], \text { para } \mathrm{v} \text { fatores de }
\end{aligned}
$$

AGI.

Obtém-se então o peso dos AGI'S por fatores, que é representado pela matriz a seguir, de modo que

$$
\begin{aligned}
& \sum_{i=1}^{m} a f_{i j}=1 \text { para qualquer } \mathrm{j}, \mathbf{e} \\
& 0 \leq a f_{i j} \leq 1:
\end{aligned}
$$$$
\left\|a f_{i j}\right\|=\left[\begin{array}{cccc}
a f_{11} & a f_{12} & \ldots & a f_{1 v} \\
a f_{21} & & & \\
\ldots & & & \\
a f_{m 1} & \ldots & \ldots & a f_{m v}
\end{array}\right] \text {, }
$$ 


\section{PRODUÇĀO}

para $\mathrm{v}$ fatores, e m AGI'S

O peso geral do AGI é obtido através da relação:

$$
\left\|a_{i}\right\|=\left\|a f_{i j}\right\| \cdot\left\|i a_{j}\right\|=\left\|\sum_{j=1}^{v} a f_{i j} i a_{j}\right\|
$$

\section{Fatores de Ponderação dos TSI'S}

Estes fatores compōem uma visão tecnológica no processo de priorização. São considerados $q$ fatores que são agregados de modo a compor um índice geral para cada TSI. Dentre os diversos fatores que podem ser agregados sāo apresentados a seguir alguns:

- Indice de Oportunidade Tecnológica - Representa as opçōes de mercado em hardware e software para suprir em diversidade e qualidade os vários Tipos de Serviços de Informaçāo.

- Índice de Impacto na Rotina de execução dos Processos - Representa o grau relativo, entre os outros TSI's, de mudanças significativas na forma de trabalho dos usuários acarretadas pela implantação do TSI.

- Índice de Custo Relativo entre os TSI's - Indica o custo relativo entre os tipos de Serviços de Informação.

Obtém-se o índice relativo dos $q$ fatores TSI, representado pelo vetor, de modo que

$$
\begin{aligned}
& \sum_{i=1}^{q} i t_{i}=1, \text { e } 0 \leq i t_{i} \leq 1: \\
& \left\|i t_{i}\right\|=\left[\begin{array}{c}
i t_{1} \\
\ldots \\
i t_{q}
\end{array}\right], \text { para q fatores de }
\end{aligned}
$$

TSI.

Obtém-se então o peso dos TSI'S por fatores, que é representado pela matriz a seguir, de modo que $\sum_{i=1}^{r} t f_{i j}=1$ para qualquer $\mathrm{j}$, e $0 \leq t f_{i j} \leq 1$ :

$$
\left\|t f_{i j}\right\|=\left[\begin{array}{cccc}
t f_{11} & t f_{12} & \ldots & t f_{1 q} \\
t f_{21} & & & \\
\ldots & & & \\
t f_{r 1} & \ldots & \ldots & t f_{r q}
\end{array}\right]
$$

para q fatores, e r TSI'S

Finalmente, obtêm-se os pesos dos TSI'S, representado pelo vetor $\left\|t_{i}\right\|=\left[\begin{array}{c}t_{1} \\ \ldots \\ t_{r}\end{array}\right]$, e obtido pela relação a 
seguir, de modo que $\sum_{i=1}^{r} t_{i}=1$, e

$0 \leq t_{i} \leq 1:$

$\left\|t_{i}\right\|=\left\|f_{i j}\right\| \cdot\left\|i_{j}\right\|$

$$
\left\|a t_{i j}\right\|=\left[\begin{array}{cccc}
a t_{11} & a t_{12} & \ldots & a t_{1 m} \\
a t_{21} & & & \\
\ldots & & & \\
a t_{r 1} & \ldots & \ldots & a t_{r m}
\end{array}\right]
$$

Através das duas matrizes anteriores

(6)

\section{Ponderação dos MSI's}

Após a obtenção dos fatores de ponderação dos AGI'S e dos fatores de ponderação dos TSI'S, determinam-se os pesos dos MSI"s. Para tal, combinam-se estes fatores, obtendo-se o peso dos MSI's com base em cada conjunto de fatores.

Inicialmente, obtêm-se os graus de participação dos TSI's em cada AGI através da matriz a seguir, considerando

$$
\begin{aligned}
& \sum_{i=1}^{r} t a_{i j}=1 \text { para qualquer } j, \quad \text { e } \\
& 0 \leq t a_{i j} \leq 1:
\end{aligned}
$$

$$
\left\|t a_{i j}\right\|=\left[\begin{array}{cccc}
t a_{11} & t a_{12} & \ldots & t a_{1 m} \\
t a_{21} & & & \\
\ldots & & & \\
t a_{r 1} & \ldots & \ldots & t a_{r m}
\end{array}\right]
$$

Igualmente obtêm-se os graus em que os AGI's usam cada TSI, através da matriz a seguir, considerando $\sum_{j=1}^{m} a t_{i j}=1$ para qualquer i, e $0 \leq a t_{i j} \leq 1$ : btêm-se os pesos dos MSI's com base nos AGI's e nos TSI's, respectivamente.

Os pesos dos MSI's com base nos AGI's são dados pela matriz a seguir, através da relação subseqüente, de modo que

$$
\begin{aligned}
\sum_{j=1}^{m} \sum_{i=1}^{r} s a_{i j}=1, \text { e } & 0 \leq s a_{i j} \leq 1: \\
\left\|s a_{i j}\right\| & =\left[\begin{array}{cccc}
s a_{11} & s a_{12} & \ldots & s a_{1 m} \\
s a_{21} & & & \\
\ldots & & & \\
s a_{r 1} & \ldots & \ldots & s a_{r m}
\end{array}\right] \\
s a_{i j} & =t a_{i j} \cdot a_{j}, \text { para qualquer i e j. }
\end{aligned}
$$

Os pesos dos MSI's com base nos TSI's são dados pela matriz a seguir, através da relação subseqüente, de modo que

$$
\sum_{j=1}^{m} \sum_{i=1}^{r} s t_{i j}=1 \text {, e } 0 \leq s t_{i j} \leq 1 \text { : }
$$

$$
\left\|s t_{i j}\right\|=\left[\begin{array}{cccc}
s t_{11} & s t_{12} & \ldots & s t_{1 m} \\
s t_{21} & & & \\
\ldots & & & \\
s t_{r 1} & \ldots & \ldots & s t_{r m}
\end{array}\right]
$$




\section{PRODUÇÃO}

$$
=\underset{i j}{s t_{i j}}=a t_{i j} \cdot t_{i} \text {, , para qualquer } \mathrm{i}, \mathrm{j}
$$

Neste estágio, pode-se combinar as duas visōes de ponderação dos MSI's, considerando os pesos relativos TSI e AGI, dados por:

- $\mathrm{pt}=$ peso da dimensão TSI

- $\quad \mathrm{pa}=$ peso da dimensão $\mathrm{AGI}$;

Considera-se $\mathrm{pt}+\mathrm{pa}=1$

Finalmente obtém-se a matriz final de pesos dos MSI's, apresentada a seguir e obtida através da relação subseqüente, de modo que $\sum_{j=1}^{m} \sum_{i=1}^{r} s_{i j}=1$, e $0 \leq s_{i j} \leq 1$ :

$$
\begin{aligned}
& \left\|s_{i j}\right\|=\left[\begin{array}{cccc}
s_{11} & s_{12} & \ldots & s_{1 m} \\
s_{21} & & & \\
\ldots & & & \\
s_{r 1} & \ldots & \ldots & s_{r m}
\end{array}\right] \\
& \left\|s_{i j}\right\|=p a\left\|s a_{i j}\right\|+p t\left\|s t_{i j}\right\|
\end{aligned}
$$

Portanto, esta matriz apresenta a visão global de ponderação dos Módulos de Sistemas de Informação (MSI's), a partir da qual é estabelecido o Plano de Ação a ser desenvolvido na organização.

Este processo de priorização é ilustrado a seguir através de um estudo de casos.

\section{Estudo de Caso}

A seguir são apresentados os resultados obtidos em cada fase da metodologia de Planejamento, sendo dado ênfase à fase de priorização. Estes resultados correspondem a um estudo de caso, baseado em um contexto de uma unidade de negócio de uma Empresa de energia elétrica e são apresentados com o intuito de ilustrar a utilização da metodologia.

$\mathrm{Na}$ etapa estratégica obtêm-se os seguintes fatores estratégicos com respectivos pesos: manter nível mínimo de fornecimento de serviços $(0,35)$, novos negócios $(0,2)$, aumentar padrão de qualidade $(0,3)$, desenvolvimento de pessoal $(0,15)$.

No contexto analisado, assumem-se os seguintes processos: Infra-Estrutura, Operaçōes, Serviços, Aquisiçāo, Desenvolvimento de Tecnologia, Gerência de Recursos Humanos, Suporte Corporativo, e Marketing.

Para o estudo de caso foram considerados os seguintes AGI's: Sistema Físico, Pessoal, Gestão de Serviços, Avaliação de Desempenho de Sistemas, e Recursos Financeiros.

Iniciando o processo de priorização, obtêm-se as informaçōes relativas ao grau de relaçāo entre os processos e os fatores estratégicos estabelecidos, conforme tabela 1. 
PRODUÇÃO

\begin{tabular}{|l|r|r|r|r|}
\hline \multicolumn{1}{|c|}{ PROCESSOS } & \multicolumn{4}{|c|}{ FATORES ESTRATÉGICOS } \\
\hline & $\begin{array}{c}\text { Aumentar| } \\
\text { serviços }\end{array}$ & $\begin{array}{c}\text { fornecer } \\
\text { negócios }\end{array}$ & \multicolumn{1}{c|}{$\begin{array}{c}\text { novos } \\
\text { qualidade }\end{array}$} & $\begin{array}{c}\text { desenvolvimento } \\
\text { de pessoal }\end{array}$ \\
\hline INFRAESTRUTURA & 0,3 & 0,2 & 0,1 & 0,05 \\
\hline OPERAÇŌES & 0,1 & 0,05 & 0,15 & 0,05 \\
\hline SERVIÇOS & 0,05 & 0,05 & 0,25 & 0,25 \\
\hline AQUISIÇĀO & 0,025 & 0,05 & 0,05 & 0,05 \\
\hline DESEN. TECNOLÓGICO & 0,2 & 0,3 & 0,1 & 0,3 \\
\hline GERÊNCIA RH & 0,1 & 0,1 & 0,15 & 0,05 \\
\hline SUPORTECORPORATIVO & 0,025 & 0,05 & 0,05 & 0,15 \\
\hline MARKETING & 0,2 & 0,2 & 0,15 & \\
\hline
\end{tabular}

Tabela 1 - Matriz $\left\|r_{i j}\right\|$

Aplicando estes valores aos pesos dos fatores estratégicos conforme a equação (1), são obtidos os índices de aderência a os fatores estratégicos para cada processo, conforme segue: Infra-estrutura $(0,18)$, Operações $(0,097)$, Serviço $(0,11)$,

Aquisição (0,04), Desenvolvimento Tecnológico $(0,205)$, Gerência de Recursos Humanos $(0,145)$, Suporte Corporativo $(0,04)$ e Marketing $(0,18)$.

O grau de relação entre AGI e processos é mostrado na tabela 2 .

\begin{tabular}{|l|r|r|r|r|r|}
\hline PROCESSOS & \multicolumn{5}{|c|}{ AGI's } \\
\hline & $\begin{array}{c}\text { Sistema } \\
\text { fisico }\end{array}$ & Pessoal & $\begin{array}{c}\text { Gestāo de } \\
\text { serviços }\end{array}$ & $\begin{array}{c}\text { Avaliação de } \\
\text { desempenho de } \\
\text { sistema }\end{array}$ & $\begin{array}{c}\text { Recursos } \\
\text { financeiros }\end{array}$ \\
\hline INFRAESTURA & 0,3 & 0,05 & 0,2 & 0,15 & 0,3 \\
\hline OPERAÇÕES & 0,25 & 0,15 & 0,3 & 0,25 & 0,05 \\
\hline SERVICOS & 0,2 & 0,1 & 0,3 & 0,3 & 0,1 \\
\hline AQUISIÇÃO & 0,4 & 0,1 & 0,1 & 0,1 & 0,3 \\
\hline DESEN.TEC & 0,4 & 0,25 & 0,1 & 0,1 & 0,15 \\
\hline GERÊNCIA RH & 0,1 & 0,35 & 0,25 & 0,2 & 0,1 \\
\hline SUP.CORP. & 0,1 & 0,3 & 0,1 & 0,1 & 0,4 \\
\hline MARKETING & 0,05 & 0,15 & 0,3 & 0,4 & 0,1 \\
\hline
\end{tabular}




\section{PRODUÇĀO}

Aplicando as equações (2), (3) e (4) obtêm-se, respectivamente os índices de aderência estratégica dos AGI's, de criticidade de automação e de comprometimento dos usuários. Os resultados relacionados a estes vetores são apresentados na Tabela 3.

\begin{tabular}{|l|c|r|r|r|r|}
\hline \multicolumn{1}{|c|}{ ÍNDICES } & \multicolumn{5}{|c|}{ AGI } \\
\hline & $\begin{array}{c}\text { Sistema } \\
\text { físico }\end{array}$ & Pessoal & $\begin{array}{c}\text { Gestão } \\
\text { de } \\
\text { serviços }\end{array}$ & $\begin{array}{c}\text { Avaliação de } \\
\text { desempenho } \\
\text { de sistema }\end{array}$ & $\begin{array}{c}\text { Recursos } \\
\text { financeiros }\end{array}$ \\
\hline $\begin{array}{l}\text { ADERENCIA } \\
\text { ESTRATÉGICA }\end{array}$ & 0,227 & 0,179 & 0,217 & 0,213 & 0,162 \\
\hline COMPR.USU. & 0,220 & 0,187 & 0,215 & 0,205 & 0,172 \\
\hline $\begin{array}{l}\text { CRITICIDADE. } \\
\text { AUTOMACAO }\end{array}$ & 0,215 & 0,175 & 0,215 & 0,205 & 0,189 \\
\hline $\begin{array}{l}\text { PESO GLOBAL } \\
\text { DO AGI }\end{array}$ & 0,222 & 0,179 & 0,216 & 0,209 & 0,174 \\
\hline
\end{tabular}

\section{Tabela 3 - Vetores $\left\|a e_{i}\right\|,\left\|a u_{i}\right\|,\left\|\alpha c_{i}\right\|,\left\|a_{i}\right\|$.}

Aplicando-se a equação (5) aos valores da Tabela $3 \mathrm{e}$ aos pesos a seguir: ie $=0,45$; ic $=0,2 ; \mathrm{iu}=0,35$, obtém-se o Peso geral dos AGI's, indicados na última linha da Tabela 3. Num procedimento similar obtêm-se os dados e aplica-se a equação
(6) visando à obtenção dos pesos para cada TSI, conforme segue: TSIT $(0,14)$, TSIG $(0,115), \operatorname{TSAD}(0,146), \operatorname{TSIE}(0,2)$, TSAE $(0,146)$, TSAP $(0,125)$, TSAG $(0,13)$.

A tabela 4 mostra $o$ grau de participação de cada TSI em cada AGI.

\begin{tabular}{|l|r|r|r|r|r|}
\hline \multirow{2}{*}{ TS I } & \multicolumn{7}{|c|}{ A GI } \\
\cline { 2 - 6 } & $\begin{array}{c}\text { Sistema } \\
\text { físico }\end{array}$ & Pessoal & $\begin{array}{c}\text { Gestāo de } \\
\text { serviços }\end{array}$ & $\begin{array}{c}\text { Avaliação de } \\
\text { desempenbo } \\
\text { de sistema }\end{array}$ & $\begin{array}{c}\text { Recursos } \\
\text { financeiro } \\
\text { s }\end{array}$ \\
\hline TSIT & 0,4 & 0,3 & 0,2 & 0 & 0,3 \\
\hline TSIG & 0,1 & 0,3 & 0,2 & 0,3 & 0,2 \\
\hline TSAD & 0 & 0,2 & 0,1 & 0,3 & 0,1 \\
\hline TSIE & 0 & 0,1 & 0 & 0,3 & 0,2 \\
\hline TSAE & 0 & 0,1 & 0 & 0,05 & 0,2 \\
\hline TSAP & 0,2 & 0 & 0,4 & 0,05 & 0 \\
\hline TSAG & 0,3 & 0 & 0,1 & 0 & 0 \\
\hline
\end{tabular}

Tabela 4 - Matriz $\left\|t a_{i j}\right\|$ 
A Tabela 5 mostra o grau em que cada AGI utiliza cada TSI.

\begin{tabular}{|c|c|c|c|c|c|}
\hline \multirow[t]{2}{*}{ TSI } & \multicolumn{5}{|c|}{ AGI } \\
\hline & $\begin{array}{l}\text { Sistema } \\
\text { físico }\end{array}$ & Pessoal & $\begin{array}{l}\text { Gestão de } \\
\text { serviços }\end{array}$ & $\begin{array}{c}\text { Avaliação de } \\
\text { desempenho } \\
\text { de sistema }\end{array}$ & $\begin{array}{l}\text { Recursos } \\
\text { financeiros }\end{array}$ \\
\hline TSIT & 0,2 & 0,2 & 0,3 & 0 & 0,3 \\
\hline TSIG & 0,1 & 0,1 & 0,2 & 0,4 & 0,2 \\
\hline TSAD & 0 & 0,15 & 0,15 & 0,4 & 0,3 \\
\hline TSIE & 0 & 0,2 & 0 & 0,45 & 0,35 \\
\hline TSAE & 0 & 0,35 & 0 & 0,1 & 0,55 \\
\hline TSAP & 0,2 & 0 & 0,7 & 0,1 & 0 \\
\hline TSAG & 0,7 & 0 & 0,3 & 0 & $\overline{0}$ \\
\hline
\end{tabular}

Aplicam-se as equaçōes (7) e (8) para se obter as matrizes correspondentes aos pesos dos MSI's nas duas visōes.

Neste estágio, deve-se combinar as duas visões de ponderação dos MSI's, considerando os pesos relativos TSI e AGI, dados por: peso da dimensão TSI, $\mathrm{pt}=0,3$; peso da dimensão $A G I$, pa $=0,7$. Observase que isto retrata o fato de que a ponderação obtida pelo AGI é mais importante, pois reflete a visão de importância dos fatores estratégicos e dos processos.

Desta forma, obtêm-se os resultados finais aplicando-se a equação (9). Estes resultados são apresentados na tabela 6 . Os valores com zero, indicam que o MSI não existe, pois para um dado $A G I$ não se aplica o correspondente TSI.

\begin{tabular}{|l|r|r|r|r|r|}
\hline TSI & \multicolumn{7}{|c|}{ AGI } \\
\hline & $\begin{array}{c}\text { Sistema } \\
\text { fisico }\end{array}$ & Pessoal & $\begin{array}{c}\text { Gestão de } \\
\text { serviços }\end{array}$ & $\begin{array}{c}\text { Avaliação de } \\
\text { desempenho de sistema }\end{array}$ & $\begin{array}{c}\text { Recursos } \\
\text { financeiros }\end{array}$ \\
\hline TSIT & $\mathbf{0 , 0 4 6}$ & $\mathbf{0 , 0 3 6}$ & $\mathbf{0 , 0 4 2}$ & 0 & 0,045 \\
\hline TSIG & 0,0147 & $\mathbf{0 , 0 2 4}$ & 0,029 & 0,050 & 0,026 \\
\hline TSAD & 0 & $\mathbf{0 , 0 2 6}$ & $\mathbf{0 , 0 2 2}$ & 0,059 & 0,035 \\
\hline TSIE & 0 & $\mathbf{0 , 0 3 3}$ & 0 & 0,081 & 0,059 \\
\hline TSAE & 0 & $\mathbf{0 , 0 4 1}$ & 0 & 0,013 & 0,066 \\
\hline TSAP & $\mathbf{0 , 0 3 0}$ & 0 & 0,087 & 0,011 & 0 \\
\hline TSAG & $\mathbf{0 , 0 8 3}$ & 0 & 0,033 & 0 & 0 \\
\hline
\end{tabular}

Tabela 6 - Matriz $\left\|s_{i j}\right\|$ 


\section{PRODUÇÃO}

O sistema de apoio a decisão desenvolvido fornece um processo interativo para obtenção dos pesos, em cada etapa, efetuando todos os cálculos necessários. Ao final o resultado é apresentado na forma de uma relação classificada em funçāo dos MSI's de maior peso, além de informaçōes gráficas.

O sistema permite a realização de análise de sensibilidade, onde se verifica, por exemplo, que a priorização não apresenta resultados sensíveis a variações do peso de 0,3 atribuído aos índices do TSI, o qual pode variar entre 0,25 e 0,35 .

\section{Comentários Finais}

O artigo apresenta uma proposta para o processo decisório na priorização de Sistemas de Informaçăo (SI) dentro de um contexto de Planejamento de SI que considera vá rios critérios, desde fatores estratégicos da organização até aspectos técnicos de ferramentas a serem utilizadas na implementação do SI.

A agregação dos diversos fatores é efetuada aplicando-se uma função valor aditiva. $O$ procedimento resulta em valores finais (pesos) dos módulos de SI a serem desenvolvidos ou incrementados, de modo a direcionar a elaboração de um Plano de Ação para o SI.

A escala obtida para ponderar os módulos de SI é de natureza ordinal. A artigo enfatiza mais a questão de agregação dos valores iniciais dos critérios do que seu processo de obtençāo. Vários aspectos poderão ser desenvolvidos em trabalhos futuros, tais como, uso de funçāo utilidade visando incorporar aspectos de incerteza associados aos fatores analisados (Almeida, 1997). Além disso, busca-se introduzir aspectos de cardinalidade na mesma, o que possibilitaria uma gradação de quanto um MSI seria mais prioritário que os outros.

Recebido em: $12 / 05 / 97$ Recebido após modificaçōes: 23/04/98 Aceito após revisão: 12/11/98

Publicado em: 25/03/99

\section{Referências Bibliográficas}

Almeida, A. T. de; Bohoris, G. A.; Steinberg, H.; (1992) Managenment Information and Decision Support System of a Telecomunication Network. Journal of Decision Systems. 1(2-3)pp.213-241.

Almeida, A. T. de; Steinberg, H.; Bohoris, G. A.; (1991) A Decision Support System in the Management of a Telecommunication Network. IFORS First Specialized Conference on Decision Support System. 27-29 March 1991; Bruges -Belgium; 4.F11.Cpp.67.

Almeida,A.T.de; Alcoforado, M.M.D.G;(1996a) O Planejamento de Sistemas de Informação na Integração da produção. Second International Congres of Industrial Engineering and XVI National Congres of Production Engineering (ENEGEP), 7-10 October, 1996, Piracicaba, SP, Brazil,

Almeida, A. T. de; Alcoforado, M.M.D.G;(1996b) Apoio a Decisāo no Planejamento e Administração de Sistemas de Informação. $20^{\circ}$ Encontro Anual da Associação Nacional dos Programas de pós-Graduação em Administração; 23-25 


\section{PRODUÇÃO}

Setembro 1996; Angra dos Reis, RJ

Davenport, T. H. (1994), Reengenharia de Processos, 1994

Sprague Jr, R. H., Watson, H. J. (Ed) (1989); Decision Support Systems - Putting Theory into Practice, Prentice-Hall, Inc; 1989

Bigdoli, H (1989), Decision Support System - Principles and Practice; West Publishing Company, New York, 1989

Thierauf, R. J.; Decision Support
System for Effective Planning and Control - A case Study Approach (1982), PrenticeHall, Inc., Englewood Cliffs, 1982

Vincke, Philippe, Multicriteria Decision-Aid (1992), John Wiley \& Sons, 1992.

Keeney, R. L.; Raiffa, H.; (1976) Decision with Multiple Objectives: Preferences and Value Trade-offs. John Wiley \& Sons

Almeida, A. T., (1997); Decision Modelling on Planning and Management of Information Systems. EURO XV INFORMS XXXIV Joint International Meeting; Barcelona, Spain, July 14-17, 1997. 